\title{
The Computational Structure of Progress Conditions
}

\author{
Gadi Taubenfeld \\ The Interdisciplinary Center, P.O.Box 167, Herzliya 46150, Israel, \\ tgadi@idc.ac.il, \\ http://www.faculty.idc.ac.il/gadi/
}

\begin{abstract}
Understanding the effect of different progress conditions on the computability of distributed systems is an important and exciting research direction. For a system with $n$ processes, we define exponentially many new progress conditions and explore their properties and strength. We cover all the known, symmetric and asymmetric, progress conditions and many new interesting conditions. Together with our technical results, the new definitions provide a deeper understanding of synchronization and concurrency.
\end{abstract}

Keywords: Progress conditions, wait-freedom, obstruction-freedom, $S$-freedom, consensus, synchronization, contention, cooperation, universality, hierarchy.

\section{Introduction}

We define exponentially many new progress conditions and explore their properties and relative strength. Our results regarding the computational structure of the new and known, symmetric and asymmetric, progress conditions provide a deeper understanding of synchronization and concurrency. Most of the known progress conditions can be classified as either cooperation-based conditions or contention-based conditions. Cooperation arises when several processes need to coordinate their actions in order to achieve a common goal. Contention arises when several processes compete for exclusive use of shared resources, such as communication bandwidth, data items or files.

Fault-freedom, the weakest cooperation-based condition, guarantees that every process will complete its pending operations provided that all the processes participate and there are no failures. Obstruction-freedom, the weakest contention-based condition, guarantees that a process will be able to complete its pending operations in a finite number of its own steps, if all the other processes "hold still" (i.e., do not take any steps) long enough [11]. Wait-freedom, the strongest both contention-based and cooperationbased progress condition, guarantees that every process will always be able to complete its pending operations in a finite number of its own steps, regardless of the behavior of the other processes [10]. While a consensus object can be implemented using only atomic registers under either fault-freedom or obstruction-freedom, it can not be implemented using registers under wait-freedom.

We start by proving two general impossibility results for symmetric progress conditions, which have many interesting implications. For example, we show that objects that satisfy cooperation-based progress conditions can be implemented from objects that satisfy the corresponding contention-based conditions, but not vice versa. We establish a formal connection between symmetric and asymmetric progress conditions, 
which enables us to apply the general impossibility results for proving new results also for asymmetric progress conditions. For the special case where only atomic registers are used, we give a complete characterization under which symmetric progress conditions consensus is solvable, and prove impossibility results for the asymmetric case. Finally, we prove a general universality result.

\subsection{Exponentially many symmetric progress conditions}

From now on we assume that the number of processes is $n$ and $n \geq 2$. A process is active when it has pending operations, otherwise it is passive. For a set of processes $P$, let $|P|$ denotes the size of $P$. For a given point in a computation, active.P is the number of active processes in $P$. We use $S$ to denote a non-empty set such that $S \subseteq\{1, \ldots, n\}$.

Definition. For any non-empty set $S$, the progress condition $S$-freedom guarantees that for every set of processes $P$, if at some point in a computation active. $P=|P|$ and $|P| \in S$, then every process in $P$ will be able to eventually complete its pending operations, provided that (1) all the processes not in $P$ do not take steps for long enough; and (2) none of the processes in $P$ fails (which means that each of the processes in $P$ will continue to take steps until it becomes passive).

Let $A$ be an algorithm for $n$ processes that satisfies $S$-freedom for some set $S$. Furthermore, assume that for some number $k, k \in S$ and $k-1 \notin S$. Assume that for a set of processes $P$ at some point in a computation of $A$, active. $P=|P|=k$, and that all the processes not in $P$ do not take any more steps. Since not all the processes in $P$ become passive at the same time eventually some process in $P$ will become passive and once this happens active. $P=k-1$. It is important to notice, that although at this point active. $P=k-1 \notin S$, the definition of $S$-freedom guarantees that (because in the near past all $k$ processes were active) eventually every process in $P$ will become passive.

It is possible to weaken the requirement that "every process in $P$ will be able to eventually complete its pending operations", and only require that "some process in $P$ will be able to eventually complete its pending operations". For one-shot objects (also called tasks), like consensus, most of our results apply also in this case.

Since the number of non-empty subsets of $\{1, \ldots, n\}$ is $2^{n}-1$, there are $2^{n}-1$ different progress conditions. They relate to known progress conditions as follows: The condition $\{n\}$-freedom is the same as fault-freedom; $\{1\}$-freedom is the same as obstruction-freedom; $\{1, \ldots, n\}$-freedom is the same as wait-freedom; $\{1, n\}$-freedom is the progress condition which implies both obstruction-freedom and fault-freedom. For $1 \leq k \leq n,\{1, \ldots, k\}$-freedom is the same as $k$-obstruction-freedom. We call these $n$ conditions, contention-based progress conditions, since $\{1, \ldots, k\}$-freedom guarantees progress under contention of at most $k$ processes. For $0 \leq t \leq n-1,\{n-t, \ldots, n\}$ freedom is the same as $t$-resiliency. We call these $n$ conditions, cooperation-based progress conditions, since $\{n-t, \ldots, n\}$-freedom captures the ability to tolerate $t$ faults.

Clearly, an object that satisfies $T$-freedom satisfies also $S$-freedom, for any $S \subset T$. For any given set $S$, we say that $S$-freedom is a symmetric progress conditions in the sense that a given process is not favored with respect to the others. 


\subsection{Asymmetric progress conditions}

The notion of asymmetric progress conditions was coined and investigated in [13]. It is motivated by the observation that some processes may be more important than others and hence should get stronger progress guarantees. Thus, an asymmetric progress condition specifics the progress guarantees for each process separately. One such progress condition which is defined in [13], called $(n, x)$-liveness, satisfies wait-freedom for $x$ processes and satisfies obstruction-freedom for the remaining $n-x$ processes.

In the literature, saying that an object is wait-free is the same as saying that each one of the processes is wait-free w.r.t. that object. Although using the term wait-freedom in two different ways may be confusing, it simplifies the discussion. Following this "tradition", we will say that an object is $S$-free iff each process is $S$-free w.r.t. that object. Clearly, a process that is $T$-free is also $S$-free, for any $S \subset T$. Asymmetric progress conditions can be practically motivated by modern multicore architectures where processes in different cores might be provided with different progress guarantees.

\subsection{Our contributions}

A consensus object $o$ supports one operation: o.propose $(v)$ satisfying: (1) Agreement: In any run, the o.propose() operation returns the same value, called the consensus value, to every process that invokes it. (2) Validity: In any run, if the consensus value is $v$, then some process invoked o.propose $(v)$. When $v \in\{0,1\}$ the object is called a binary consensus object. By a consensus object we mean a binary consensus object; and by $n$-consensus we mean a multi-valued consensus object where $v \in\{0,1, \ldots, n-1\}$. The term register means an atomic read/write register. A simple (but not obvious) observation is that, for two positive integers $m$ and $n$, and a set $S \subseteq\{1, \ldots, \min (m, n)\}$, it is not always possible to implement an $S$-free consensus object for $n$ processes using $S$-free consensus objects for $m$ processes and registers. Our results are:

New Definitions. We define exponentially many progress conditions and investigate their properties and relative strength. Together with the technical results, the new notions provide a deeper understanding of synchronization and concurrency.

General impossibility results. Let $S$ be a subset of $\{1, \ldots, n\}$, where $n \geq 2$. $|S|$ is the number of elements in $S$, and $\max . S$ and $\min . S$ are the largest and the smallest elements in $S$, respectively. The width of $S$, denoted width.S, is defined as follows: width.S $=1+\max . S-\min . S$. We prove the following two impossibility results,

- For any set $|S| \geq 2$, it is not possible to implement an $S$-free consensus object for $n$ processes using any number of wait-free consensus objects for width. $S-1$ processes and registers.

- For any two sets $S$ and $T$, and integer $k$, if $|T| \geq 2, k \in T, k \notin S$ and $k \leq$ width.T then it is not possible to implement a $T$-free consensus object for $n$ processes using any number of $S$-free consensus objects for $n$ processes and registers.

It follows from the results that: (1) for any $2 \leq k \leq n$, it is not possible to implement a $\{1, k\}$-free consensus object for $k$ processes using any number of wait-free consensus 
objects for $n-1$ processes and registers; and (2) For any $n>2$, it is not possible to implement a wait-free consensus object for two processes using any number of $\{1, n\}$ free consensus objects for $n$ processes and registers.

Cooperation vs. contention. It follows from the above impossibility results that objects which satisfy cooperation-based progress conditions can not be used to implement objects which satisfy contention-based progress conditions. However, objects that satisfy cooperation-based conditions can be implemented from objects that satisfy the corresponding contention-based conditions. More formally,

- It is not possible to implement $\{1,2\}$-free consensus object for $n$ processes using $\{2, \ldots, n\}$-free consensus objects for $n$ processes and registers. However, for $2 \leq$ $k \leq n$, it is possible to implement an $\{n-k+1, \ldots, n\}$-free consensus object for $n$ processes using $\{1, \ldots, k\}$-free consensus objects for $n$ processes and registers.

This result is rather surprising, given the fact that while cooperation-based conditions imply fault-freedom, contention-based conditions do not imply the fault-freedom.

Asymmetric progress conditions. We establish a connection between symmetric and asymmetric conditions, which enables us to apply the general impossibility results for proving new results also for asymmetric conditions. For example, we show that:

- For any two integers $k_{1}$ and $k_{2}$ such that $1 \leq k_{1}<k_{2} \leq n$, it is not possible to implement a consensus object for $n$ processes that satisfies $\left\{k_{1}, k_{2}\right\}$-freedom for $n-k_{2}+1$ processes and satisfies $\left\{k_{1}\right\}$-freedom for all the other processes, using any number of wait-free consensus objects for $k_{2}-k_{1}$ processes and registers.

Atomic registers. When only registers are used, we have a complete characterization under which symmetric conditions consensus is solvable, and prove impossibility results for the asymmetric case. For the symmetric case, we show that:

- For any set $S$, it is possible to implement an $S$-free consensus object for $n$ processes using registers if and only if $|S|=1$.

The results generalize the famous FLP result for the case of one faulty process $[6,18]$.

Universality. We generalize results regarding the universality of consensus from [10]. An object $o$ is $S$-universal for $n$ processes if any object which has sequential specification has an $S$-free linearizable implementation using registers and objects of type $o$ for $n$ processes. We prove that,

- For any positive integer $n$, and any non-empty set $S \subseteq\{1, \ldots, n\}$, an $S$-free consensus object for $n$ processes is $S$-universal for $n$ processes.

The result implies that an object $o$ is $S$-universal for $n$ processes if and only if an $S$ free consensus object for $n$ processes can be implemented from objects of type $o$ and registers. The wait-free hierarchy [10], is an infinite hierarchy of objects, such that the objects at level $i$ are exactly those objects which are $\{1, \ldots, i\}$-universal for $i$ processes, but are not $\{1, \ldots, i+1\}$-universal for $i+1$ processes. We will explain, how to define other interesting hierarchies. 


\subsection{Related work}

The consensus problem was formally defined in [20]. The impossibility result that there is no consensus algorithm that can tolerate even a single crash failure in an asynchronous model was first proved for the message-passing model in [6], and later has been extended for the shared memory model in which only atomic registers are supported, in [18]. A recent survey which covers many related impossibility results can be found in [4]. The power of shared objects has been studied extensively in environments where processes may fail benignly, and where every operation is wait-free. In [10], Herlihy classified objects by their consensus numbers and defined the wait-free hierarchy. Additional results regarding the wait-free hierarchy can be found in $[14,16]$.

Objects that can be used, together with registers, to build wait-free implementations of any other object are called universal objects. Previous work provided methods, called universal constructions, to transform sequential specifications of arbitrary shared objects into wait-free concurrent implementations that use universal objects [10,21]. In [21] it is proved that sticky bits are universal, and independently, in [10] it is proved that wait-free consensus objects are universal. A bounded space version of the universal construction from [10] appears in [15]. Linearizability is defined in [12].

Two extensively studied conditions are wait-freedom [10] and obstruction-freedom [11]. It is shown in [11] that obstruction-free consensus is solvable using registers. Various contention management techniques have been proposed to improve obstructionfreedom under contention [7, 22]. Other works investigated boosting obstruction-freedom by making timing assumption $[1,5]$ and using failure detectors [8]. Wait-free consensus algorithms that use registers in the absence of contention and revert to using strong synchronization operations when contention occurs, are presented in $[2,17,19]$.

The notion of asymmetric progress conditions was coined in [13], where the $(n, x)$ liveness condition which guarantees wait-freedom for $x$ processes and obstructionfreedom for the remaining $n-x$ processes, was defined. The following results are proven in [13]: (1) It is not possible to implement an $(n, 1)$-live consensus object using wait-free consensus objects for $n-1$ processes and registers; (2) For $1 \leq x<n-1$, an $(n, x)$-live consensus object is strictly weaker than an $(n, x+1)$-live consensus object, thereby establishing a hierarchy for $(n, x)$-liveness; (3) It is not possible to implement a consensus object for $n$ processes which guarantees both fault-freedom and obstruction-freedom for one process and only obstruction-freedom for the remaining $n-1$ processes, using wait-free consensus objects for $n-1$ processes and registers; (4) It is possible to implement a consensus object for $n \geq x$ processes that satisfies a condition called asymmetric group-based progress condition using $(x, x)$-live consensus objects and registers.

The notion of $k$-obstruction-freedom is presented in [24], as part of a transformation that is used to fuse objects which avoid locking and locks together in order to create new types of shared objects. In [25], a new classification for evaluating the strength of shared objects is proposed. The classification is based on finding, for each object of type $o$, the largest $k$ for which it is possible to solve consensus for any number processes, using any number of objects of type $o$ and registers, assuming that the required progress condition is $k$-obstruction-freedom. The main technical result in [25] is that the new classification is equivalent to Herlihy's traditional classification. 
Although progress conditions and adversaries are two seemingly different notions, they are actually closely related. In [3], a precise way is presented to characterize adversaries by introducing the notion of disagreement power: the biggest integer $k$ for which the adversary can prevent processes from agreeing on $k$ values when using registers only; and it is shown how to compute the disagreement power of an adversary. Our formalism for expressing progress conditions is not expressive enough to express all the adversaries considered in [3], and vice versa. In the last section, we generalize our formalism to express both.

\section{Preliminaries}

Our model of computation consists of an asynchronous collection of $n$ processes that communicate via shared objects. An event corresponds to an atomic step performed by a process. For example, the events which correspond to accessing registers are classified into two types: read events which may not change the state of the register, and write events which update the state of a register but does not return a value. We use the notation $e_{p}$ to denote an instance of an arbitrary event at a process $p$.

A run is a pair $(f, R)$ where $f$ is a function which assigns initial states (values) to the objects and $R$ is a finite or infinite sequence of events. An implementation of an object from a set of other objects, consists of a non-empty set $C$ of runs, a set $N$ of processes, and a set of shared objects $O$. For any event $e_{p}$ at a process $p$ in any run in $C$, the object accessed in $e_{p}$ must be in $O$. Let $x=(f, R)$ and $x^{\prime}=\left(f^{\prime}, R^{\prime}\right)$ be runs. Run $x^{\prime}$ is a prefix of $x$ (and $x$ is an extension of $x^{\prime}$ ), denoted $x^{\prime} \leq x$, if $R^{\prime}$ is a prefix of $R$ and $f=f^{\prime}$. When $x^{\prime} \leq x,\left(x-x^{\prime}\right)$ denotes the suffix of $R$ obtained by removing $R^{\prime}$ from $R$. Let $R$; T be the sequence obtained by concatenating the finite sequence $R$ and the sequence $T$. Then $x ; T$ is an abbreviation for $(f, R ; T)$.

Process $p$ is enabled at run $x$ if there exists an event $e_{p}$ such that $x ; e_{p}$ is a run. For simplicity, we write $x p$ to denote either $x ; e_{p}$ when $p$ is enabled in $x$, or $x$ when $p$ is not enabled in $x$. Register $r$ is a local register of $p$ if only $p$ can access $r$. For any sequence $R$, let $R_{p}$ be the subsequence of $R$ containing all events in $R$ which involve $p$. Runs $(f, R)$ and $\left(f^{\prime}, R^{\prime}\right)$ are indistinguishable for a set of processes $P$, denoted by $(f, R)[P]\left(f^{\prime}, R^{\prime}\right)$, iff for all $p \in P, R_{p}=R_{p}^{\prime}$ and $f(r)=f^{\prime}(r)$ for every local register $r$ of $p$. When $P=\{p\}$ we write $[p]$ instead of $[P]$. It is assumed that the processes are deterministic, that is, if $x ; e_{p}$ and $x ; e_{p}^{\prime}$ are runs then $e_{p}=e_{p}^{\prime}$.

The runs of an asynchronous implementation of an object must satisfy several properties. For example, if a write event which involves $p$ is enabled at run $x$, then the same event is enabled at any finite run that is indistinguishable to $p$ from $x$. In the following proofs, we will implicitly make use of few such straightforward properties.

\section{Impossibility results}

We use $S$ and $T$ to denote non-empty sets which are subsets of $\{1, \ldots, n\} ;|S|$ is the number of elements in $S$, and $\max . S$ and min.S are the largest and the smallest elements in $S$, respectively. The width of $S$, denoted width.S, is defined as follows: 
width. $S=1+\max . S-\min . S$. Thus, the width of the set $\{1, \ldots, n\}$ is $n$. We notice that it is always the case that width. $S \geq|S|$.

Theorem 1. For any set $|S| \geq 2$, it is not possible to implement an $S$-free consensus object for $n$ processes using any number of wait-free consensus objects for width.S -1 processes and registers.

It follows immediately from Theorem 1 that for any $2 \leq k \leq n$, it is not possible to implement a $\{1, k\}$-free consensus object for $n$ processes using any number of waitfree consensus objects for $k-1$ processes and registers. Next we consider the relative strength of different condition for the same number of processes.

Theorem 2. For any two sets $S$ and $T$, and integer $k$, if $|T| \geq 2, k \in T, k \notin S$ and $k \leq$ width. $T$ then it is not possible to implement a $T$-free consensus object for $n$ processes using any number of $S$-free consensus objects for $n$ processes and registers.

It follows from Theorem 2 that: For any $n>2$, it is not possible to implement a waitfree consensus object for two processes using any number of $\{1, n\}$-free consensus objects for $n$ processes and registers. Next we prove the theorems. For lack of space, the proofs of all the lemmas appear only in the full version. (The full version of the paper can be downloaded from: www.faculty.idc.ac.il/gadi .)

\subsection{A detailed proof}

The proofs of Theorem 1 and Theorem 2 use the following notions, abbreviations, and lemmas. Let $N$ be the set of all $n$ processes, and let $P \subseteq N$. A finite run $x$ is $(P, v)$ valent if in all extensions of $x$, by processes in $P$ only, where a decision is made, the decision value is $v(v \in\{0,1\})$. A run is $P$-univalent if it is either $(P, 0)$-valent or $(P, 1)$-valent, otherwise it is $P$-bivalent. We say that two $P$-univalent runs are $P$ compatible if they have the same valency, that is, either both runs are $(P, 0)$-valent or both are $(P, 1)$-valent. Finally, we say that process $p \in P$ is a $P$-decider at run $x$ if for every extension $y$ of $x$ by steps of processes from $P$ only (i.e. $x[N-P] y$ ), the run $y p$ is $P$-univalent. Recall that we assume that $S \subseteq\{1, \ldots, n\}$.

Lemma 1. Let $|S| \geq 2$, and let $P$ be a set of processes such that $|P|=\max . S$. Then, for every $p \in P$, there is at least one subset of $P$, denoted $p . S P$, of size min.S which does not include $p$.

Lemma 2. For a set $S$ and non-empty sets of processes $P$ and $Q$ such that $|P| \in S$, $|Q| \in S$ and $Q \subseteq P$, in any $S$-free consensus object, if two $P$-univalent runs are indistinguishable for $Q$ and the state of all the objects that (processes in) $Q$ can access are the same at these runs, then these runs must be P-compatible.

Lemma 3. Let $|S| \geq 2$ and let $P$ be a set of processes such that $|P|=\max . S$. Then, every $S$-free consensus object has a P-bivalent empty run.

Lemma 4. Let $|S| \geq 2$ and let $P$ be a set of processes such that $|P|=$ max.S. Let $y$ be a run of an $S$-free consensus object, and let $p \in P$ and $q \in P$ be two different processes such that (1) $y \neq y p$ and $y \neq y q$, (2) the runs yp and yqp are $P$-univalent and not $P$-compatible. Then, in their two next events from $y, p$ and $q$ are accessing the same object, and this object is not a register. 
Lemma 5. Let $|S| \geq 2$ and let $P$ be a set of processes such that $|P|=$ max.S. For every $S$-free consensus object there is a P-bivalent run $x$ and process $p \in P$ such that $p$ is a P-decider at $x$.

Lemma 6. Let $|S| \geq 2$ and let $P$ be a set of processes such that $|P|=$ max.S. Every $S$-free consensus object has a $P$-bivalent run y and two processes $p \in P$ and $q \in P$ such that: (1) $p$ is a P-decider at $y$; (2) the runs yp and yqp are $P$-univalent and not $P$-compatible; and (3) in their two next events from $y, p$ and $q$ are accessing the same object, and this object is not a register.

Lemma 7. Let $|S| \geq 2$ and let $P$ be a set of processes such that $|P|=$ max.S. Every $S$-free consensus object has a $P$-bivalent run $y$, a set $Q \subseteq P$ of size width.S, and two processes $p \in Q$ and $q \in Q$ such that: (1) $p$ is a P-decider at $y$; (2) the runs yp and yqp are $P$-univalent and not $P$-compatible; and (3) in their next events from $y$, all the width.S processes in $Q$, are accessing the same object, and this object is not a register.

Proof of Theorem 1. It follows from Lemma 7 that every implementation of an $S$-free consensus object for $n$ processes, must use an object, say $o$, which at least width.S processes must be able to access at the same run, and $o$ is not a register. Thus, it is not possible to implement an $S$-free consensus object for $n$ processes using any number of wait-free consensus objects for width.S -1 processes and registers.

Proof of Theorem 2. Assume to the contrary that there is such an implementation of a $T$-free consensus object for $n$ processes from $S$-free consensus objects for $n$ processes and registers. Let $P$ be a set of processes such that $|P|=\max$. $T$. It follows from Lemma 7 that such an implementation has a $P$-bivalent run $y$, a set $Q \subseteq P$ of size width.T, and two processes $p \in Q$ and $q \in Q$ such that: (1) $p$ is a $P$-decider at $y$; (2) the runs yp and yqp are $P$-univalent and not $P$-compatible; and (3) in their next events from $y$, all the width.S processes in $Q$, are accessing the same object, say $o$, and this object is not a register. Thus, it must be the case that $o$ is an $S$-free consensus object.

Assume that at the end of $y$, just before the width.T processes access $o, n-k$ processes fail and the remaining $k$ active processes, including $p$ and $q$, are about to access $o$. Since there are only $k$ active processes and $k \in T$, the implementation of a $T$-free consensus object must guarantee that these $k$ processes will eventually properly terminate. However, since $k \notin S$, the $S$-free consensus object $o$ does not guarantee that any of the remaining $k$ active processes will ever get a response from $o$. Assume none of the $k$ processes ever gets a response for $o$. Although the $k$ processes may continue to take steps, because $y p$ and $y q p$ are $P$-univalent and not $P$-compatible, the final decision value (of the $T$-free consensus object) depends on getting a response from $o$. Without a response from $o$, it is not possible to determine whether the prefix of the current run is yp or yqp. Thus, the $k$ processes will never be able to terminate. A contradiction.

\section{Cooperation vs. contention}

It follows from the impossibility results that objects which satisfy cooperation-based progress conditions can not implement objects which satisfy contention-based progress conditions. More formally, 
Theorem 3. It is not possible to implement $\{1,2\}$-free consensus object for $n$ processes using $\{2, \ldots, n\}$-free consensus objects for $n$ processes and registers.

Proof. Let $T$ be the set $\{1,2\}$, and $k=1$. Then, (1) $|T| \geq 2$, (2) $k \in T$, (3) $k \notin$ $\{2, \ldots, n\}$, and (4) $k \leq$ width. T. Thus, the result follows from Theorem 2 .

Next we show that objects that satisfy cooperation-based conditions can be implemented from objects that satisfy the corresponding contention-based conditions.

Theorem 4. For $2 \leq k \leq n$, it is possible to implement an $\{n-k+1, \ldots, n\}$-free consensus object for $n$ processes using $\{1, \ldots, k\}$-free consensus objects for $n$ processes and registers.

To prove theorem 4, we first generalize a known result for wait-freedom, namely, that multi-valued consensus can be implemented from binary consensus ([23], page 329).

Lemma 8. For any $k \geq 2, n \geq 2$ and $S \subseteq\{1, \ldots, n\}$, an $S$-free $k$-consensus object for $n$ processes can be implemented from $S$-free binary consensus objects for $n$ processes and atomic bits.

Proof. To implement a singe $k$-consensus object, we use $\lceil\log k\rceil$ binary consensus objects, which are numbered 0 through $\lceil\log k\rceil-1$, and $k$ bits which are numbered 0 through $k-1$ and are initialized to 0 . To propose a value $v \in\{0, \ldots, k-1\}, p$ does the following: (1) it sets the bit number $v$ to 1; (2) it proposes the binary encoding of $v$, bit by bit, to the binary consensus objects in an increasing order starting from number 0 . If at some point during the second step the bit $p$ has proposed is not accepted as the consensus value at the corresponding binary consensus object, $p$ stops proposing $v$, scan the bits and chooses one of the bits that are set to 1 , say $v^{\prime}$, which also matches the values that has successfully proposed so far and continues to propose the value $v^{\prime}$. This procedure continues until $p$ proposes, to all the $\lceil\log k\rceil$ binary consensus objects. The value that its binary encoding was successfully proposed to all the $\lceil\log k\rceil$ binary consensus objects is the final consensus value.

Proof of Theorem 4: Build a tree of degree $k$ with $\lceil n / k\rceil$ leaves, and where each node of the tree is a $\{1, \ldots, k\}$-free $k$-consensus object. Each participating process is progressing from a leaf to the root, where at each level of the tree it accesses a $k$-consensus object, competing against at most $k-1$ processes in its neighbor's subtree. As a process advances towards the root, it plays the role of process 0 (i.e., proposes 0 ) when it arrives from the left most subtree, of process $k-1$ when it arrives from the right most subtree, or of process $0 \leq i \leq k-1$ when it arrives from the $i$ 'th subtree. The winner at each node is the process its value is being agreed upon. Only a winner at a given node continues to progress towards the root. The value agreed at the root is the final decision value. Each of the processes that accesses the root writes the final decision value at a special register called decision, and decides on that value. Each process that loses at some node other than the root, spins on the decision register until a value is written into it and decides on that value. 


\section{Asymmetric progress conditions}

As already mentioned, the notion of asymmetric progress conditions was coined and investigated in [13]. Let $A P C$ be an Asymmetric Progress Condition; we define max.APC, min.APC and width.APC as follows,

- max.APC is the largest $1 \leq k \leq n$ such that (at least) $n-k+1$ processes are $\{k\}$-free, or 0 if no such $k$ exists.

- min.APC is the smallest $1 \leq k \leq n$ such that every process is $\{k\}$-free, or 0 if no such $k$ exists.

- width. $A P C$ equals $1+\max . A P C-\min . A P C$ if $\min . A P C \neq 0$, or 0 otherwise.

Thus, for the asymmetric progress condition ( $n, 1)$-liveness (as defined in [13]), $\max .(n, 1)$ liveness $=n$, min. $(n, 1)$-liveness $=1$ and width.( $n, 1)$-liveness $=n$.

Lemma 9. Let $O$ be a consensus object for $n$ processes that satisfies an asymmetric progress condition APC such that min. APC $\geq 1$. Using $O$ and a single register it is possible to implement a consensus object for $n$ processes that satisfies the symmetric progress condition (min.APC, max.APC)-freedom.

Proof. Let decision be a register which is initially set to -1 . Each process tries to reach a decision by accessing $O$. A process that reaches a decision writes the decision value into decision and terminates. Each process infinitely often reads decision, and if the value read is different from -1 , it decides on that value and terminates. Since every subset of $\max . A P C$ processes includes at least one $\{\max . A P C\}$-free process, this implementation clearly satisfies (min.APC, max.APC)-freedom. Another way to view this implementation is: once $O$ returns a value to some processes, it keeps this value in an internal private register, and thereafter returns it immediately to every process that accesses it.

Lemma 10. Let $A P C$ be a an asymmetric progress condition such that $1 \leq \min . A P C<$ $\max . A P C \leq n$, and let $P$ be a set of processes such that $|P|=\max . A P C$. Every consensus object for $n$ processes that satisfies APC has a $P$-bivalent run $y$, a set $Q \subseteq P$ of size width. $A P C$, and two processes $p \in Q$ and $q \in Q$ such that: (1) $p$ is a $P$-decider at $y$; (2) the runs yp and yqp are $P$-univalent and not $P$-compatible; and (3) in their next events from $y$, all the width.APC processes in $Q$, are accessing the same object, and this object is not a register.

Proof. Assume to the contrary that $O$ is a consensus object for $n$ processes that satisfies $A P C$, and $O$ does not have a run $y$ with all the three properties as mentioned in Lemma 10. By Lemma 9, using $O$ and a single register it is possible to implement a consensus objects $O^{\prime}$ for $n$ processes that satisfies the symmetric progress condition (min.APC, max.APC)-freedom. Thus, also $O^{\prime}$ does not have such a run $y$. However, this contradicts Lemma 7.

Theorem 5. For any asymmetric progress condition APC such that $1 \leq$ min. APC $<$ max.APC $\leq n$, it is not possible to implement a consensus object for $n$ processes that satisfies APC using any number of wait-free consensus objects for width.APC -1 processes and registers. 
Proof. The proof is similar to that of Theorem 1. It follows from Lemma 10 that every implementation of a consensus object for $n$ processes that satisfies $A P C$, must use an object, say $o$, which at least width. APC processes must be able to access at the same run, and $o$ is not a register. Thus, it is not possible to implement a consensus object for $n$ processes that satisfies APC using any number of wait-free consensus objects for width. $A P C-1$ processes and registers.

It is proven in [13] that it is not possible to implement an $(n, 1)$-live consensus object using any number of wait-free consensus objects for $n-1$ processes and registers; and that this result holds even when the requirement that one process should be waitfree is replaced with the much weaker requirement that one process is $\{1, n\}$-free. These important results are special cases of the following corollary of Theorem 5.

Corollary 1. For any two positive integers $k_{1}$ and $k_{2}$ such that $1 \leq k_{1}<k_{2} \leq n$, it is not possible to implement a consensus object for $n$ processes, that satisfies $\left\{k_{1}, k_{2}\right\}$ freedom for $n-k_{2}+1$ processes and satisfies $\left\{k_{1}\right\}$-freedom for all the other processes, using any number of wait-free consensus objects for $k_{2}-k_{1}$ processes and registers.

Another interesting result from [13] is that: For $1 \leq x<n-1$, an $(n, x)$-live consensus object is strictly weaker than an $(n, x+1)$-live consensus object, thereby establishing a hierarchy for $(n, x)$-liveness. Using Lemma 10 it is possible to slightly generalize this result.

\section{Atomic registers}

For the case where only registers are used, we present a complete characterization under which symmetric progress conditions consensus is solvable, and prove impossibility results for the asymmetric case.

\section{Theorem 6.}

- For any set $S$, it is possible to implement an $S$-free consensus object for $n$ processes using registers if and only if $|S|=1$.

- For any asymmetric progress condition APC, it is not possible to implement a consensus object for $n$ processes that satisfies APC using registers if width.APC $>1$.

Proof. It follows from Theorem 1 that it is not possible to implement an $S$-free consensus object for $n$ processes using registers if $|S| \geq 2$, and it follows from Theorem 5 that it is not possible to implement a consensus object for $n$ processes that satisfies APC using registers if width. $A P C>1$.

Next, we show that for any integer $1 \leq k \leq n$, it is possible to implement a $\{k\}$-free consensus object for $n$ processes using registers. The algorithm (i.e., implementation) proceeds in rounds. The notion of a round is used only for the sake of describing the algorithm. We do not assume a synchronous model of execution in which all the processes are always executing the same round.

Each process has a preference for the decision value in each round; initially this preference is the input value of the process. If no decision is made in a round then the processes advance to the next round, and try again to reach agreement. 
IMPLEMENTING $\{k\}$-FREE CONSENSUS FOR $n$ PROCESSES USING REGISTERS (WHERE $k \in$ $\{1, \ldots, n\}$ ): program for process $p_{i}$ with input $i n_{i}$ (where $i n_{i} \in\{0,1\}$ and $i \in\{1, \ldots, n\})$.

\section{shared registers}

$x[0 . . \infty, 0 . .1]$ infinite array of bits, initially $x[0,0]=x[0,1]=1$ and all other entries are 0 flag $[1 . . \infty, 1 . . n]$ infinite array of bits, initially all entries are 0 decide ranges over $\{\perp, 0,1\}$, initially $\perp$

\section{local registers}

$r_{i}$ integer, initially 1

$v_{i}$ bit, initially $i n_{i} ; l_{i}$, count $_{i}$ integers, initial values are immaterial

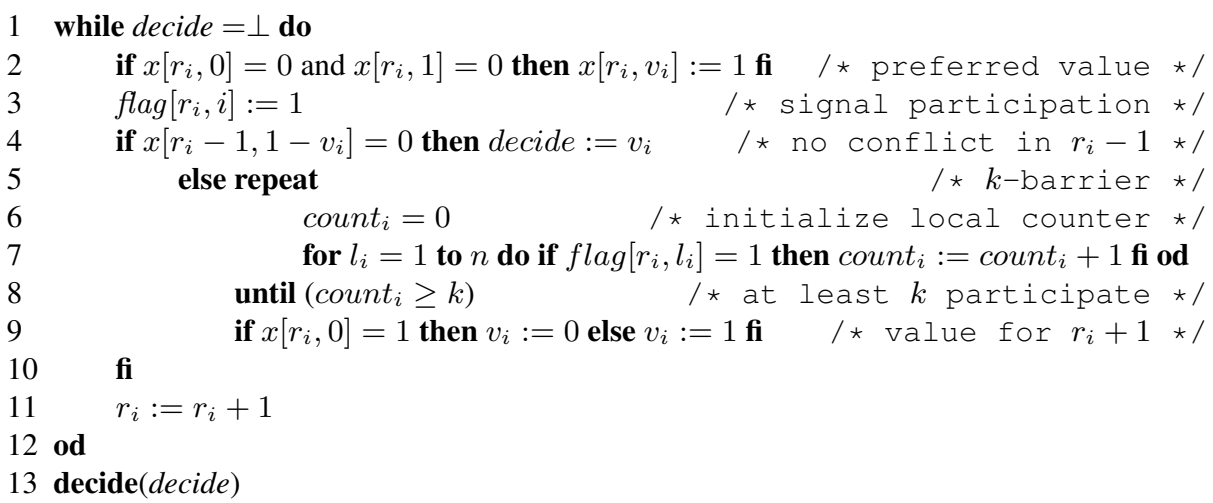

In round $r \geq 1$, process $p_{i}$ first checks if the bit of its preference $v_{i}$ and of the opposite value $1-v_{i}$ are set. If both bits are not set, $p_{i}$ sets its preference bit $v_{i}$ by writing 1 to $x\left[r, v_{i}\right]$ (line 2). Then, $p_{i}$ sets its participation bit by writing 1 to $f l a g\left[r_{i}, i\right]$ (line 3 ). Next, $p_{i}$ reads the bit $x\left[r-1,1-v_{i}\right]$. If the bit $x\left[r-1,1-v_{i}\right]$ is not set, then every process that reaches round $r$ with the conflicting preference $1-v_{i}$ will find that only $x\left[r, v_{i}\right]$ is set to 1 , will never set $x\left[r, 1-v_{i}\right]$ to 1 . Consequently, process $p_{i}$ can safely decide on $v_{i}$, and it writes $v_{i}$ to decide (line 4). Otherwise, waits until it notices that at least $k$ processes are participating in round $r$ (lines 5-8). After that $p_{i}$ updates its preference in an attempt to agree with the other processes (line 9). Then, $p_{i}$ proceeds to round $r+1$ (line 11).

If exactly $k$ processes with possibly conflicting preferences participate in round $r$, then they will reach line 9, only after all of them set their flags in line 3 . This implies that once some process reaches line 9 , no process is at line 2 , and hence all the $k$ processes will reach round $r+1$ with the same preference which is the value chosen in line 9 . When all processes reach a round with the same preference, a decision is reached either in that round or the next round.

\section{Universality}

In [10], the notion of universality is introduced in the context of wait-freedom. An object $o$ is (wait-free) universal for $n$ processes if any object which has sequential specification 
has a wait-free linearizable implementation using registers and objects of type $o$ in a system with $n$ processes. Below we generalize the notion of wait-free universality.

Definition. An object $o$ is $S$-universal for $n$ processes if any object which has sequential specification has an $S$-free linearizable implementation using registers and objects of type $o$ for $n$ processes.

One of the important results proved in [10], is that wait-free consensus for $n$ processes is universal for $n$ processes. Next we generalize this result.

Theorem 7. For any positive integer $n$, and any non-empty set $S \subseteq\{1, \ldots, n\}$, an $S$ free consensus object for $n$ processes is $S$-universal for $n$ processes.

To prove the result, we present a universal construction that implements any $S$-free object $o$ for $n$ processes from $S$-free consensus objects for $S$ processes and registers. The construction conceptually mimics the original construction for the wait-free model from [10]. In the full version of the paper, we give such a construction, which is similar to the one for the wait-free model from [23]. A similar type of a universality result (with a similar proof) can be proved also for asymmetric progress conditions.

Corollary 2. For any object o, any positive integer $n$, and any non-empty set $S \subseteq$ $\{1, \ldots, n\}, o$ is $S$-universal for $n$ processes if and only if an $S$-free consensus object for $n$ processes can be implemented from objects of type o and registers.

The wait-free hierarchy is an infinite hierarchy of objects, introduced in [10], such that the objects at level $i$ of the hierarchy are exactly those objects which are $\{1, \ldots, i\}$ universal for $i$ processes, but are not $\{1, \ldots, i+1\}$-universal for $i+1$ processes. For that hierarchy, by the above definition, (1) no object at level less than $i$ together with registers can implement any object at level $i$; and (2) each object at level $i$ together with registers can implement any object at level $i$ or at a lower level.

The wait-free hierarchy is meaningful because it can be defined using only the (contention-based) progress conditions $\{1, \ldots, k\}$-freedom, for all $k$. In such a case, there is a total order, based on the stronger than relation, between all these conditions. Similar such hierarchies, in which there is a total order between the conditions, can be naturally defined. For example, by using the cooperation-based progress conditions, the cooperation hierarchy can be defined as follows: For a given system of $n$ processes, the objects at level $i$ of the hierarchy are exactly those objects which are $\{n-i+1, \ldots, n\}$ universal for $n$ processes, but are not $\{n-i, \ldots, n\}$-universal for $n$ processes.

\section{Discussion}

It is possible to extend the definitions of progress conditions in various ways. Below we define two such new interesting extensions.

Definition. For any non-empty set $S \subseteq\{1, \ldots, n\}$ and an integer $1 \leq k \leq n$, the progress condition $(S, k)$-freedom guarantees that for every set of processes $P$, if at some point in a computation active. $P=|P|$ and $|P| \in S$, then (at least) $\min \{k,|P|\}$ processes in $P$ will be able to eventually complete their pending operations, provided 
that (1) all the processes not in $P$ do not take steps for long enough; and (2) none of the processes in $P$ fails.

We notice that in a system of $n$ processes, $(S, n)$-freedom is the same as $S$-freedom; and $(\{1, \ldots, n\}, 1)$-freedom is the same as a known condition called non-blocking [12] (sometimes also called lock-freedom).

Definition. Let $W_{1}, \ldots, W_{n}$ be sets of sets of process identifiers such $P \in W_{i}$ only if $p_{i} \in P$. The progress condition $\left(W_{1}, \ldots, W_{n}\right)$-freedom guarantees that for every set of processes $P$ and every process $p_{i}$, if at some point in a computation active. $P=|P|$ and $P \in W_{i}$, then process $p_{i}$ will be able to eventually complete its pending operations, provided that (1) all the processes not in $P$ do not take steps for long enough; and (2) none of the processes in $P$ fails.

Each one of the adversaries considered in [3] corresponds to some $\left(W_{1}, \ldots, W_{n}\right)$ free progress condition, which has the following property: For every set $P$, if $P \in W_{i}$ and $p_{j} \in P$ then $P \in W_{j}$. We notice that satisfying this property, completely precludes the ability to express the asymmetric progress conditions defined in the introduction. That is, w.r.t. this definition, this property distinguishes between symmetric and asymmetric progress conditions (adversaries).

Additional interesting questions are: exploring the complexity and computability of problems like set-consensus, renaming, etc. under various new progress conditions; exploring the relation to failure detectors, by possibly extending known results for waitfreedom [9]; defining meaningful hierarchies; better understanding of the relations between different progress conditions; adding timing assumptions.

Known open problems, like the robustness of the wait-free hierarchy or whether a queue object can be implemented from a set of test-and-set objects, fetch-and-add objects, swap objects and atomic registers, for $n \geq 3$, can now be studied in our more general setting.

The study should not be limited to shared memory systems only. Consider for example $n$ senders that are trying to broadcast the same message to a single receiver, and it is required that at least one of the senders succeeds to transmit, without collisions, whenever an odd number of senders broadcast at the same time. This required progress condition, and similar ones, that are sometimes expressed using the notion of a conflict graph, can be easily formally expressed and studied within our general framework.

\section{References}

1. M. K. Aguilera and S. Toueg. Timeliness-based wait-freedom: a gracefully degrading progress condition. In Proc. 27rd ACM Symp. on Principles of Distributed Computing, pages 305-314, 2008.

2. H. Attiya, R. Guerraoui, and P. Kouznetsov. Computing with reads and writes in the absence of step contention. Proceedings of the 19th International Symposium on Distributed Computing, LNCS 3724, 122-136, 2005.

3. C. Delporte-Gallet, H. Fauconnier, R. Guerraoui, and A. Tielmann. The disagreement power of an adversary. Proc. of the 23rd International Symp. on Distributed Computing, Spain, September 2009. LNCS 5805, 8-21, 2009.

4. F. E. Fich and E. Ruppert. Hundreds of impossibility results for distributed computing. Distributed Computing, 16(2-3):121-163, 2003. 
5. E. F. Fich, V. Luchangco, M. Moir, and N. Shavit. Obstruction-free algorithms can be practically wait-free. Proc. of the 19th International Symp. on Distributed Computing, LNCS 3724, pp. 78-92, 2005.

6. M.J. Fischer, N.A. Lynch, and M.S. Paterson. Impossibility of distributed consensus with one faulty process. Journal of the ACM, 32(2):374-382, 1985.

7. R. Guerraoui, M. P. Herlihy and B. Pochon. Towards a theory of transactional contention managers. In Proc. of the 24th Symp. on Principles of Dist. Computing, pp. 258-264, 2005.

8. R. Guerraoui, M. Kapalka and P. Kouznetsov. The weakest failure detectors to boost obstruction-freedom. Distributed Computing, 20(6):415-433, 2008.

9. R. Guerraoui and P. Kouznetsov. Failure detectors as type boosters. Distributed Computing, 20:343-358, 2008.

10. M. P. Herlihy. Wait-free synchronization. ACM Trans. on Programming Languages and Systems, 13(1):124-149, January 1991.

11. M. P. Herlihy, V. Luchangco, and M. Moir. Obstruction-free synchronization: Double-ended queues as an example. In Proc. of the 23rd Int. Conf. on Dist. Computing Systems, 2003.

12. M. P. Herlihy and J. M. Wing. Linearizability: a correctness condition for concurrent objects. TOPLAS, 12(3):463-492, 1990.

13. D. Imbs, M. Raynal, and G. Taubenfeld. On asymmetric progress conditions. In Proc. 29th ACM Symp. on Principles of Distributed Computing, 2010. To appear.

14. P. Jayanti. Robust wait-free hierarchies. Journal of the ACM, 44(4):592-614, 1997.

15. P. Jayanti and S. Toueg. Some results on the impossibility, universality, and decidability of consensus. In Proc. of the 6th Int. Workshop on Distributed Algorithms: LNCS 674, pages 69-84, 1992.

16. Wai-Kau Lo and Vassos Hadzilacos. All of us are smarter than any of us: Nondeterministic wait-free hierarchies are not robust. SIAM Journal on Computing, 30(3):689-728, 2000.

17. V. Luchangco, M. Moir and N. Shavit. On the uncontended complexity of consensus. Proc. of the 17th International Symp. on Distributed Computing, LNCS 2848, 45-59, 2003.

18. M.C. Loui and H. Abu-Amara. Memory requirements for agreement among unreliable asynchronous processes. Advances in Computing Research, 4:163-183, 1987.

19. M. Merritt and G. Taubenfeld. Resilient consensus for infinitely many processes. Proc. of the 17th International Symp. on Distributed Computing, LNCS 2848, 1-15, 2003.

20. M. Pease, R. Shostak, and L. Lamport. Reaching agreement in the presence of faults. Journal of the ACM, 27(2):228-234, 1980.

21. S. A. Plotkin. Sticky bits and universality of consensus. In Proc. 8th ACM Symp. on Principles of Distributed Computing, pages 159-175, 1989.

22. W. N. Scherer and M. L. Scott. Advanced contention management for dynamic software transactional memory. In Proc. of the 24th Symp. on Principles of Dist. Computing, pp. 240-248, 2005.

23. G. Taubenfeld. Synchronization Algorithms and Concurrent Programming. Pearson / Prentice-Hall, ISBN 0-131-97259-6, 423 pages, 2006.

24. G. Taubenfeld. Contention-sensitive data structures and algorithms. Proc. of the 23rd International Symp. on Distributed Computing, Spain, 2009. LNCS 5805 , 157-171, 2009.

25. G. Taubenfeld. On the computational power of shared objects. Proc. of the 13th international conf. on principles of distributed systems, France, 2009. LNCS 5923 , 270-284, 2009. 